\title{
ISCEV Standard for full-field clinical electroretinography (2008 update)
}

\author{
M. F. Marmor · A. B. Fulton · G. E. Holder · Y. Miyake • M. Brigell • \\ M. Bach (for the International Society for Clinical Electrophysiology of Vision)
}

Received: 24 October 2008/Accepted: 29 October 2008/Published online: 22 November 2008

(C) Springer-Verlag 2008

\begin{abstract}
This document, from the International Society for Clinical Electrophysiology of Vision (ISCEV), presents an updated and revised ISCEV
\end{abstract}

This document was approved by ISCEV on 15 July, 2008 at the Annual General Meeting in Morgantown, WV, USA. This document is available on the ISCEV website: http://www. iscev.org.

The authors represent the International Society for Clinical Electrophysiology of Vision.

M. F. Marmor ( $\square)$

Department of Ophthalmology, Stanford University

School of Medicine, Stanford, CA, USA

e-mail: marmor@stanford.edu

\section{A. B. Fulton}

Department of Ophthalmology, Children's Hospital,

Harvard Medical School, Boston, MA, USA

G. E. Holder

Department of Electrophysiology, Moorfields Eye

Hospital, London, UK

Y. Miyake (Chair)

Division of Orthoptics and Visual Science,

Aichi Shukutoku University, Nagoya, Japan

M. Brigell

Translational Medicine, Novartis Institutes for

Biomedical Research, Cambridge, MA, USA

M. Bach

Univeritäts-Augenklinik, Freiburg, Germany
Standard for clinical electroretinography (ERG). The parameters for flash stimulation and background adaptation have been tightened, and responses renamed to indicate the flash strength (in $\mathrm{cd} \cdot \mathrm{s} \cdot \mathrm{m}^{-2}$ ). The ISCEV Standard specifies five responses: (1) Dark-adapted 0.01 ERG (rod response); (2) Darkadapted 3.0 ERG (combined rod-cone response); (3) Dark-adapted 3.0 oscillatory potentials; (4) Lightadapted 3.0 ERG (cone response); (5) Light-adapted 3.0 flicker $(30 \mathrm{~Hz}$ flicker). An additional Darkadapted 10.0 ERG or Dark-adapted 30.0 ERG response is recommended.

Keywords Clinical standards $\cdot$ Electroretinogram

\section{Abbreviations \\ ERG \\ ISCEV

\author{
Electroretinogram \\ International Society for Clinical \\ Electrophysiology of Vision
}

\section{Introduction}

The full-field electroretinogram (ERG) is a widely used electrophysiologic test of retinal function. In 1989, ISCEV standardized basic protocols so that comparable ERGs could be recorded throughout the world [1]. Because of advances in ERG knowledge and techniques, this standard is reviewed regularly. This document supersedes the 2004 version [2]. In this revision, we define background light and stimulus flash levels as single values, rather than ranges, 
to improve the consistency of ERG responses under the Standard. As in the 2004 Standard, we recommend an additional ERG to a stronger flash for the dark-adapted eye. We also propose a new format for specifying (naming) ISCEV Standard ERGs that will make reporting clearer.

The ERG Standardization Committee recognizes that older ERG recording equipment may not comply with some stimulus parameters, but hopes that this issue will be resolved over time. Laboratories that do not fully comply with this current ISCEV Standard should indicate clearly in all reports and publications where their technique has differed.

An ISCEV Standard ERG includes the following responses, named according to conditions of adaptation and the stimulus (flash strength in $\mathrm{cd} \cdot \mathrm{s} \cdot \mathrm{m}^{-2}$ ).

(1) Dark-adapted 0.01 ERG (formerly "rod response");

(2) Dark-adapted 3.0 ERG (formerly "maximal or standard combined rod-cone response");

(3) Dark-adapted 3.0 oscillatory potentials (formerly "oscillatory potentials");

(4) Light-adapted 3.0 ERG (formerly "single-flash cone response");

(5) Light-adapted 3.0 flicker ERG (formerly "30 Hz flicker").

Recommended additional response: either Darkadapted 10.0 ERG or Dark-adapted 30.0 ERG.

The five basic ERGs represent the minimum ERG evaluation for clinical diagnosis. This standard describes simple technical procedures that allow reproducible recordings from most patients. Different procedures can provide comparable ERGs. It is incumbent upon users of alternative techniques to demonstrate that their procedures do in fact produce signals that are equivalent in basic waveform, amplitude, and physiological significance to the Standard.

It is intended that ISCEV Standard ERG protocols be used widely, but not to the exclusion of other tests or protocols that are not covered by this standard (see Table 1). We encourage electrophysiologists to extend test protocols to maximize the diagnostic value of the ERG for their patients and for clinical trials. ISCEV has also published guidelines for calibration of electrophysiologic equipment [3], guidelines for recording the multifocal ERG [4], and standards for the pattern ERG [5], electrooculogram [6], and visual evoked potential [7].
Table 1 Examples of specialized types of ERG (not covered by this ISCEV standard)

Macular or focal ERG

Multifocal ERG (see published guidelines [4])

Pattern ERG (see published standard [5])

Early receptor potential (ERP)

Scotopic threshold response (STR)

Direct-current ERG

Long-duration light-adapted ERG (on-off responses)

Double-flash ERG

Chromatic stimulus ERG (including S-cone ERG)

Dark and light adaptation of the ERG

Dark-adapted and light-adapted luminance-response analyses

Saturated $a$-wave slope analysis

Specialized procedures for young and premature infants

Recommendations for extended protocols are in preparation. We recommend that commercial recording equipment have the capability of recording ERGs under conditions that are outside the present standard but that are nevertheless either widely used or likely to be needed in the future. This document is not a safety standard, and does not mandate particular procedures for individual patients.

The organization of this report is as follows:

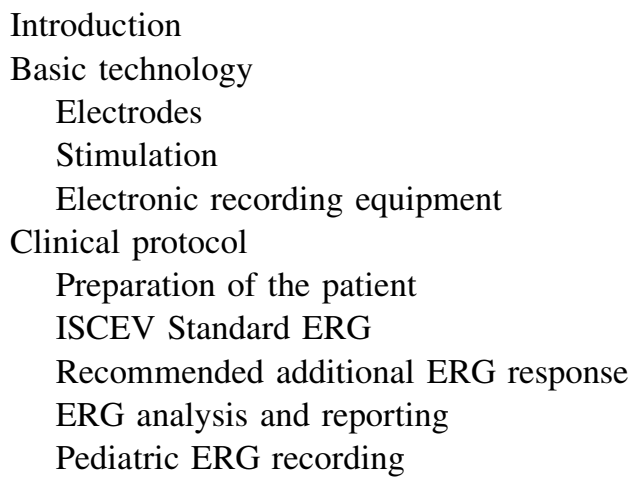

\section{Basic technology}

Electrodes

\section{Recording electrodes}

Electrodes that contact the cornea or nearby bulbar conjunctiva should be used for Standard full-field recording. These include contact lens electrodes, 
conductive fibers and foils, conjunctival loop electrodes, and corneal wicks. Contact lens electrodes will provide the highest amplitude and most stable recordings; such electrodes should be centrally transparent with an optical opening as large as possible, and incorporate a device to hold the lids apart. The corneal surface should be protected during use with a non-irritating and non-allergenic ionic conductive solution that is relatively non-viscous (i.e., no more viscous than $0.5 \%$ methyl cellulose). More viscous solutions can attenuate signal amplitude. Users should be aware that signal amplitude might be reduced with other types of corneal and conjunctival electrodes as the point of ocular contact moves away from the cornea apex. Topical anesthesia is necessary for contact lens electrodes but may not be required for other types of corneal and conjunctival electrodes. It is necessary that all electrophysiologists master the technical requirements of their chosen electrode, to ensure good ocular contact, to ensure proper electrode impedance, to ensure that waveforms are comparable to standard ERGs, and to define both normal values and variability (which may be different with different electrodes) for their own laboratory. ERGs recorded with the active electrode on the skin have low amplitudes and higher noise levels. These do not meet the ISCEV standard but may provide useful information in certain clinical situations.

\section{Reference electrodes}

Reference electrodes may be incorporated into the contact lens-speculum assembly to make contact with the conjunctiva ("bipolar electrodes"). This is the most stable configuration electrically. Alternatively, electrodes can be placed near each orbital rim, temporal to the eye as a reference for the corresponding eye. The forehead is not ideal as a reference site because of risk of signal contamination by ocular cross-over or from cortical evoked potentials. Use of other reference positions deviate from this Standard.

\section{Ground electrodes}

A separate skin electrode should be attached to an indifferent point and connected to ground. Typical locations are on the forehead or ear.
Skin reference electrode characteristics

The skin should be prepared by cleaning, and a suitable conductive paste or gel used to ensure good electrical connection. Skin electrodes used for reference or ground should have $5 \mathrm{k} \Omega$ or less impedance measured between 10 and $100 \mathrm{~Hz}$ [3]. If more than one skin electrode is used (e.g., for reference and ground) they should all have similar impedance.

\section{Electrode stability}

The baseline voltage in the absence of light stimulation should be stable. Some reference electrode systems may need to be made of non-polarized material to achieve this stability.

\section{Electrode cleaning}

Recording the ERG involves the exposure of corneal electrodes to tears and potential exposure of the skin electrodes to blood if there is any abrasion of the skin surface. Electrodes (if not disposable) must be suitably cleaned and sterilized after each use to prevent transmission of infectious agents. The cleaning protocol should follow manufacturers' recommendations and current national standards for devices that contact skin and tears.

Stimulation

\section{Light diffusion}

Full-field (Ganzfeld) stimulation should be used. With focal flashes, the area of retinal illumination is not uniform, and its extent is unknown. Full-field dome stimulators are generally preferable to ocular diffusers (such as 100-diopter or opalescent contact lenses) because it is difficult with the latter to measure the luminance, extent, and uniformity of the stimulus. It is incumbent on manufacturers and users of diffusers to verify that full-field stimulation meets the requirements of this standard.

\section{Stimulus duration}

The standard is based on flash stimuli with durations that are considerably shorter than the integration time 
of any photoreceptor. Thus, stimuli should consist of flashes having a maximum duration of $5 \mathrm{~ms}$.

\section{Stimulus wavelength}

Flash stimuli should have a color temperature near $7,000 \mathrm{~K}$, and they should be used with domes or diffusers that are visibly white. Colored filters can be used to enhance the separation of rod and cone ERGs, but this is not part of the standard. ${ }^{1}$

\section{Stimulus strength}

Flash stimuli are quantified as time-integrated luminance at the surface of the Ganzfeld bowl. In physical terms, this represents luminous energy per unit solid angle (steradian) per unit area, which should be measured in photopic candela-seconds per meter squared $\left(\mathrm{cd} \cdot \mathrm{s} \cdot \mathrm{m}^{-2}\right){ }^{2}$

This ISCEV Standard specifies the stimulus strength for eliciting each standard ERG response, to minimize response variation. To account for minor variability in equipment and calibration, a tolerance of $\pm 10 \%$ (0.05 log unit) is acceptable within the standard. The flash stimuli and lightadapting background ISCEV Standard ERGs are as follows:

(1) For rod stimulation: $0.01 \mathrm{~cd} \cdot \mathrm{s} \cdot \mathrm{m}^{-2}$;

\footnotetext{
${ }^{1}$ Chromatic stimuli offer certain advantages in the separation of cone and rod ERGs, but the calibration of colored stimuli and the relation of the ERGs produced by them to the standard ERG require special procedures. White flashes should be used for the standard ERGs, whether or not other stimuli are used in addition.

${ }^{2}$ White stimuli produced by a combination of narrow band sources, such as red, green, and blue light-emitting diodes (LEDs), may not be equivalent to broad-band white light as a stimulus for both rods and cones. Manufacturers must ensure that appropriate photopic and scotopic filters are incorporated into their stimulation and calibration systems so that stimulus output is equivalent to the standard for all conditions. Separate scotopic calibration may be necessary for LED systems, and if so the proper stimulus for eliciting rod ERGs will be $2.5 \mathrm{log}$ units below a scotopically-calibrated standard flash. The word 'intensity' is widely used to describe the luminance of surfaces. However, in photometry, 'intensity' quantifies the light from a point source. Luminance is the appropriate term for extended sources such as those used for ERG stimuli and backgrounds.
}

(2) For all other standard responses: $3.0 \mathrm{~cd} \cdot \mathrm{s} \cdot \mathrm{m}^{-2}$ (this flash was formerly designated as the "Standard Flash");

(3) Light adaptation and background luminance: $30 \mathrm{~cd} \cdot \mathrm{m}^{-2}$.

For the recommended additional response: use either 10.0 or $30.0 \mathrm{~cd} \cdot \mathrm{s} \cdot \mathrm{m}^{-2}$ (laboratories should indicate the chosen value, and name the response accordingly).

\section{Nomenclature}

Stimulus (and response) names are described by the state of light adaptation, and the flash strength in $\mathrm{cd} \cdot \mathrm{s} \cdot \mathrm{m}^{-2}$. For example, the dark-adapted response to $3.0 \mathrm{~cd} \cdot \mathrm{s} \cdot \mathrm{m}^{-2}$ is called the "Dark-adapted 3.0 ERG." In addition, descriptive terms (such as "rod response," "mixed rod-cone response," etc) may be used. This scheme of naming should also apply to non-standard stimuli, which might be used for special protocols or because of equipment limitations (e.g., if flashes of $15.0 \mathrm{~cd} \cdot \mathrm{s} \cdot \mathrm{m}^{-2}$ are used under dark-adapted conditions they should be specified as a "Darkadapted 15.0 ERG”).

\section{Background illumination}

In addition to producing flashes, the stimulator must be capable of producing a steady and even background luminance across the full field, for light adaptation. A white background of $30 \mathrm{~cd} \cdot \mathrm{m}^{-2}$ should be used $( \pm 10 \%$ allowance for variations in calibration) for this standard. A chromatic background may also be used for special purposes in non-standard ERGs.

\section{Adjustment of stimulus strength and background luminance}

A method for modifying both the stimulus and background is required. Stimulation systems should be capable of producing flashes over a range of at least $3 \log$ units in strength, either continuously or in steps of not more than $0.3 \log$ unit. The method of attenuation should not change the wavelength composition of either the flash or the background light. We recognize that the stimulus and background requirements for a full range of other ERG tests will 
be more extensive, and we recommend that equipment manufacturers exceed this minimum standard. ${ }^{3}$

\section{Stimulus and background calibration}

The strength of each flash within the full-field stimulus dome must be documented by the user or manufacturer, with an integrating photometer placed at the location of the eye. The photometer must meet international standards for photometric measurements based on the photopic luminous efficiency function (photopic luminosity curve), and must be capable of recording the total output of very brief flashes. For most stroboscopic stimulators, light output per flash varies with the flash repetition rate; therefore, separate calibrations will need to be made for single and repetitive stimuli. The background luminance of the dome's surface is calibrated in a non-integrating mode. Users should consult the current ISCEV guidelines for calibration of electrophysiologic equipment [3] for a more detailed treatment of calibration procedures. We recommend that manufacturers of stimulators supply a suitable photometer with their equipment.

\section{Recalibration}

See the ISCEV guidelines for calibration [3]. Light output from a dome varies with time from changes in the flash tubes, power sources, line voltage, the background lights (particularly if they are incandescent), attenuation systems, or reflectance of the dome. Responsibility for electronic stability and warnings about sources of instability should rest with the manufacturers of the equipment. The frequency with

\footnotetext{
3 We recommend that the flash source of commercial instruments be capable of generating strengths at least $2 \log$ units above the basic $3.0-\mathrm{cd} \cdot \mathrm{s} \cdot \mathrm{m}^{-2}$ flash and be attenuable through 6 $\log$ units below that same flash. Regardless of whether attenuation is achieved by filters or electronic means, we also strongly recommend that commercial units incorporate a means of inserting additional colored and neutral density filters. These capabilities will allow electrophysiologists to perform a variety of useful protocols beyond the Standard, and will meet possible future changes in the Standard. We also suggest that background luminance be adjustable to perform electro-oculography with the same equipment. Commercial units should also allow the insertion of colored and neutral density filters into the background illumination system to meet a variety of needs.
}

which recalibration of flashes and backgrounds is required will vary from system to system and could be as high as weekly for some units. Self-calibrating units are encouraged.

\section{Electronic recording equipment}

\section{Amplification}

The bandpass of the amplifier and preamplifiers should include at least the range from 0.3 to $300 \mathrm{~Hz}$ and be adjustable for oscillatory potential recordings and special requirements. The input impedance of the preamplifiers should be at least $10 \mathrm{M} \Omega$. Amplifiers should generally be AC (alternating current) coupled (i.e., capacitatively coupled) and capable of handling offset potentials that may be produced by the electrodes. ${ }^{4}$

\section{Patient isolation}

The patient should be electrically isolated according to current standards for safety of clinical biologic recording systems in the user's country.

\section{Display of data}

The final record should represent, without attenuation, the full amplifier bandpass. Good resolution can be achieved with computer-aided (digital) systems or oscilloscopes but not with direct pen recorders. To avoid a loss of information, digitizers should sample ERGs at a rate of $1 \mathrm{kHz}$ or higher in each channel. With computer-aided systems, it is important that ERG waveforms be displayed promptly so that the operator can continuously monitor stability and make adjustments during the test procedure. Recording units that digitize ERG signals can usually average them as well. All single flash responses should be presented with at least $20 \mathrm{~ms}$ of baseline preceding the flash, to allow judgment of baseline stability. It is recommended that each type of stimulus be replicated at least once and that both responses be displayed to show consistency.

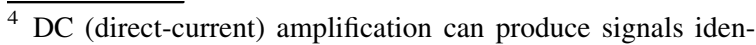
tical to those from AC amplification, but it is extremely difficult to use because of drift in baseline and offset potentials; we strongly advise AC recording except for laboratories with special requirements and expertise.
} 
Averaging

Averaging is not typically required to record quantifiable ERGs with the recommended types of electrodes. However, averaging a limited number of ERGs may decrease variability and background noise, if present. Averaging may also be used to identify and measure low amplitude pathologic ERGs. Artifact rejection must be a part of any averaging system. The time between signal repetitions should not be less than the recommendation in the standard for each type of ERG response.

\section{Clinical protocol}

Preparation of the patient

\section{Pupillary dilatation}

The pupils should be maximally dilated and the pupil size noted for all ERG recordings in this standard.

\section{Pre-adaptation to light or dark}

The recording conditions outlined below specify 20 min of dark adaptation before recording darkadapted ERGs, and 10 min of light adaptation before recording light-adapted ERGs. The choice of whether to begin with dark-adapted or light-adapted conditions is up to the user, provided these adaptation requirements are met. If contact lens electrodes are used, the wearing time can be minimized by dark adapting first, and inserting the electrodes under dim red light at the end of the adaptation period. Care should be used to avoid too bright a red light, and an additional 5 min of dark adaptation may be needed for recovery after lens insertion.

\section{Pre-exposure to light}

We advise that fluorescein angiography or fundus photography be avoided before ERG testing, but if these examinations have been performed, the subject should have at least 30 min recovery time in ordinary room illumination before beginning ERG dark adaptation. It is usually preferable to record dark-adapted ERGs to weak flashes before the ERGs to stronger flashes, to minimize the risk of light adaptation during the dark-adapted testing.

\section{Fixation}

A fixation point should be incorporated into stimulus domes. A stable eye is important so that eye movements do not alter the corneal electrode position, produce electrical artifacts, or allow blockage of light by the electrode or eyelid. Patients who cannot see a fixation target may be instructed to look straight ahead and keep their eyes steady. Patients should be monitored to assess compliance, and account for difficulties in eye opening or fixation.

\section{ISCEV standard ERG (see Fig. 1)}

\section{Dark-adapted 0.01 ERG (rod response)}

Dark-adapt the patient for a minimum of $20 \mathrm{~min}$ before recording the dark-adapted ERG (and longer if the patient had been exposed to unusually bright light). The Dark-adapted 0.01 ERG is normally the first signal measured after dark adaptation, because it is the most sensitive to light adaptation. The stimulus is a dim white flash of $0.01 \mathrm{~cd} \cdot \mathrm{s} \cdot \mathrm{m}^{-2}$; with a minimum interval of $2 \mathrm{~s}$ between flashes.

\section{Dark-adapted 3.0 ERG (combined rod-cone response)}

This is produced by a white $3.0 \mathrm{~cd} \cdot \mathrm{s} \cdot \mathrm{m}^{-2}$ flash in the dark-adapted eye. There should be an interval of at least $10 \mathrm{~s}$ between stimuli.

\section{Dark-adapted 3.0 oscillatory potentials}

Dark-adapted oscillatory potentials should be obtained from the dark-adapted eye, using the $3.0 \mathrm{~cd} \cdot \mathrm{s} \cdot \mathrm{m}^{-2}$ flash stimulus. Oscillatory potentials may also be recorded from the light-adapted eye (Light-adapted 3.0 oscillatory potentials). The high-pass filter must be set at $75-100 \mathrm{~Hz}$, and the low pass filter set at $300 \mathrm{~Hz}$ or above. Users should be aware that there are several types of electronic and digital filters, which may have different effects upon physiological signals (e.g., phase shifts or ringing). More information about filter selection and use is presented in the ISCEV guidelines for calibration [3]. 
Fig. 1 Diagram of the five basic ERG responses defined by the Standard. These waveforms are exemplary only, and are not intended to indicate minimum, maximum or even average values. Large arrowheads indicate the stimulus flash. Dotted arrows exemplify how to measure time-to-peak $(t$, implicit time), $a$-wave amplitude and $b$-wave amplitude

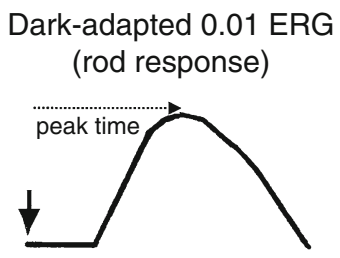

Light-adapted 3.0 ERG (cone response)

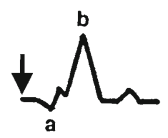

Dark-adapted 3.0 ERG (combined rod-cone response)

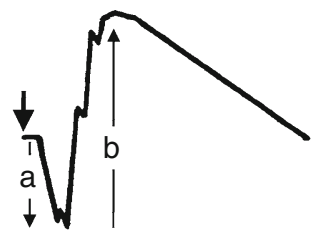

Light-adapted 3.0 flicker

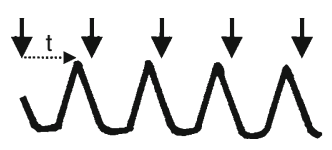

Dark-adapted 3.0

oscillatory potentials

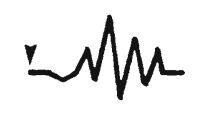

The oscillatory potentials vary with stimulus repetition rate and change after the first stimulus. For standard dark-adapted oscillatory potentials, stimulus flashes should be given $15 \mathrm{~s}$ apart; for light-adapted oscillatory potentials they would be given $1.5 \mathrm{~s}$ apart. Only the second or subsequent waveforms be retained or averaged.

\section{Light-adapted 3.0 ERG (single-flash cone response)}

Use a $3.0-\mathrm{cd} \cdot \mathrm{s} \cdot \mathrm{m}^{-2}$ stimulus, with at least $0.5 \mathrm{~s}$ between flashes. To achieve stable and reproducible cone ERGs, a minimum of 10 min light adaptation is required, because the cone ERG may increase during this period. The background luminance should be $30 \mathrm{~cd} \cdot \mathrm{m}^{-2}$ measured at the surface of the full-field stimulus bowl.

\section{Light-adapted 3.0 flicker ERG (30 Hz flicker)}

Flicker ERGs also reflect activity of the cone system, and should be obtained with $3.0-\mathrm{cd} \cdot \mathrm{s} \cdot \mathrm{m}^{-2}$ stimuli, under the same conditions of light-adaptation as the Light-adapted 3.0 ERG. Recording the flicker ERG in the light-adapted state reduces discomfort and allows the light adaptation to be standardized. Flashes should be presented at a rate of approximately 30 stimuli per second $(30 \mathrm{~Hz})$, and the rate that is chosen should be constant for the laboratory. The first ERG response to the flickering stimulus will be a singleflash waveform; thus, the first few waveforms should be discarded so that stable conditions are reached.

Some flash tubes do not produce full output while flickering, and separate calibration or a change in light attenuation may be needed to conform to the standard.

Recommended additional ERG (see Fig. 2)

\section{Dark-adapted 10.0 ERG or dark-adapted 30.0 ERG}

An additional dark-adapted ERG to a stronger flash is recommended for routine testing. This stimulus gives a larger $a$-wave with better definition (no double trough), larger oscillatory potentials that are easier to characterize, and more distinctive features of negative ERG waveforms (for critical recognition of diseases with related $b$-wave reduction). Also this stronger flash may give better signals in patients with opaque media or immature retinae. We suggest a flash strength of either 10.0 or $30.0 \mathrm{~cd} \cdot \mathrm{s} \cdot \mathrm{m}^{-2}$. Both of these values are widely used, but there is insufficient evidence as yet to choose between them. This additional ERG response should ordinarily be

Dark-adapted 10.0 ERG

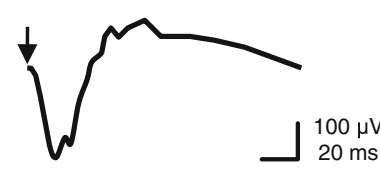

Fig. 2 Diagram of the recommended additional stronger flash ERG, either at 10.0 (left) or $30.0 \mathrm{~cd} \cdot \mathrm{s} \cdot \mathrm{m}^{-2}$ (right). Arrows indicate the time of the stimulus flash
Dark-adapted 30.0 ERG

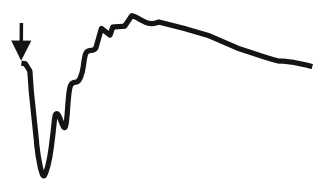


recorded after the Dark-adapted 3.0 ERG is recorded from the dark-adapted eye, with an interval of at least $20 \mathrm{~s}$ between stimuli. This response should be described according to the exact stimulus value used, e.g. "Dark-adapted 10.0 ERG", or "Dark-adapted 30.0 ERG." Note that these stronger flashes are not recommended as an addition to the light-adapted ERG, because a stronger flash may reduce the $b$-wave amplitude due to the photopic hill phenomenon.

ERG analysis and reporting

\section{Single flash ERGs}

In general, $b$-wave amplitude and time-to-peak (implicit time) is measured for all ERGs (except oscillatory potentials), and the $a$-wave should also be measured when recognizable as a distinct component. According to current convention, the $a$-wave amplitude is measured from baseline to $a$-wave trough, the $b$-wave amplitude is measured from $a$-wave trough to $b$-wave peak; the $a$-wave and $b$-wave implicit times are measured from the time of the flash to the peak of the wave (see Fig. 1).

\section{Oscillatory potentials}

There is considerable debate regarding how to measure and describe oscillatory potentials. ${ }^{5}$ Their appearance is highly dependent upon adaptation state and filter characteristics of the amplifier, but there are usually three major peaks often followed by a fourth smaller one. Simply observing the presence of the three peaks, and their normality relative to the standards of the laboratory, may be adequate for many clinical purposes in keeping with our present state of knowledge.

\section{Flicker ERG}

The amplitude of flicker ERG is measured from the trough to the peak (averaging several typical

\footnotetext{
5 An overall index of oscillatory potential amplitude can be obtained by adding up measurements of the three major peaks, preferably from lines spanning the bases of the adjacent troughs, but alternatively from adjacent troughs directly (to allow use of measuring cursors with digitized systems). Some authors advise measurement of individual peaks.
}

responses). The implicit time is measured from each stimulus onset to the corresponding peak.

\section{Normal values}

We recommend that each laboratory establish or confirm normal values for its own equipment and patient population giving attention to an appropriate sample size. Because some ERG parameters (such as $b$-wave amplitude) are not necessarily normally distributed, calculations of standard deviation may be misleading. To describe the limits of normal, the median value (not the mean) should be used, and the actual values on either side of the median that bracket $95 \%$ of the normal range of ERGs (in other words, the $95 \%$ percentile determined by direct tabulation of ERGs).

All ERG reporting (whether for local records, publication, or even for non-standard ERGs) should include normal values that show the limits of normal. Some manufactures distribute norms for their standard protocols, and several large series have been recently published that give normative data. However, ERG norms for amplitude may have to be scaled up or down depending on where the users' electrode rests on cornea or conjunctiva. Note that ERG parameters increase rapidly during infancy and decrease modestly with age thereafter. At elderly ages, the fall in amplitude can be substantial. Thus, normative values should be adjusted for age. Although circadian variations of the ERG are small under ordinary recording conditions, we recommend that the time of ERG recording be noted on all records because it could become relevant for certain diseases or for repeat measurements.

\section{Reporting the ERG}

Standardization of ERG reporting is critical to the goal of having comparable data worldwide. ERG reports should include representative waveforms of each of the standard ERGs displayed with amplitude and time calibrations and labeled with stimulus variables and the state of light or dark adaptation. These should include at least $20 \mathrm{~ms}$ of baseline prior to the stimulus for single-flash responses and, where feasible, should indicate the stimulus time for each flash with a marker or line (for flicker as well as single-flash). Two responses from each stimulus 
condition should be displayed to demonstrate the degree of consistency or variability. The timeintegrated luminance of the stimulus flashes $\left(\mathrm{cd} \cdot \mathrm{s} \cdot \mathrm{m}^{-2}\right)$ and the background luminance $\left(\mathrm{cd} \cdot \mathrm{m}^{-2}\right)$ should be given in absolute values. Reports should indicate whether the techniques of recording meet this current ISCEV standard, and any deviation should be described explicitly. All reports should give patient results listed along with normal values and ranges. Finally, reports should note the time of testing, pupil diameters, and any conditions that are not specified by the standard, including type and position of electrode, sedation or anesthesia, and the level of compliance.

\section{Pediatric ERG recording}

The ERG can be recorded from infants and young children. The younger the infant, the more chance that adult norms will not apply. Somewhat lower amplitudes and longer implicit times generally apply below 6-12 months of age under dark-adapted conditions, and below 2-3 months of age under lightadapted conditions. Below 6 months of age, the Dark-adapted 3.0 ERG may be poorly defined in healthy infants; the Dark-adapted 10.0 or 30.0 ERG is usually well defined in all infants. In general, very young or premature infants pose special problems and require special recording protocols and norms outside of this Standard.

Most pediatric patients can be studied without sedation or general anesthesia. Small infants can be swaddled if necessary. Noncompliant children (especially ages 2-6 for whom containment can be difficult) may become compliant with oral sedation. Medical guidelines should be followed with respect to indications, risks, medical monitoring requirements, and the choice of a sedative/relaxant versus general anesthesia. Considering the variability of pediatric records, there will generally be little effect on ERG amplitude or waveform with sedation or partial anesthesia, although full anesthesia may modify the ERG.

Contact lens electrodes are suitable for infants and young children, but pediatric sizes will be required with eyelid specula. Other types of corneal and skin electrodes vary in their applicability to children; greater comfort is often offset by greater electrode movement or smaller response amplitude. Special care is required with children to monitor electrode position and compliance to minimize artifacts.

The ERG matures during infancy, and newborn and infant signals must be interpreted with great caution. Later in infancy and childhood, ERGs approach adult waveform and amplitude. Pediatric ERGs should ideally be compared to those from normal subjects of the same age, although there may be little normative data available. Because movement and poor fixation can make pediatric records variable, several repetitions of each ERG should be recorded in order to recognize reproducible waveforms and choose the best examples. Standard protocols may occasionally need to be abbreviated in order to obtain the ERGs most critical to the diagnostic question under investigation. Stronger flashes may help to reveal poorly developed ERGs. Reports should note the degree of cooperation and any medications used.

Acknowledgments We thank D. L. McCulloch, J. G. Robson, and E. Zrenner for their contributions to this document.

\section{References}

1. Marmor MF, Arden GB, Nilsson SE, Zrenner E (1989) Standard for clinical electroretinography. Arch Ophthalmol 107:816-819

2. Marmor MF, Holder GE, Seeliger NW, Yamamoto S (2004) Standard for clinical electroretinography (2003 update). Doc Ophthalmol 108:107-114. doi:10.1023/B:DOOP.00000367 93.44912.45

3. Brigell M, Bach M, Barber C, Moskowitz A, Robson J (2003) Guidelines for calibration of stimulus and recording parameters used in clinical electrophysiology of vision (revised 2002). Doc Ophthalmol 107:185-193. doi:10.1023/ A: 1026244901657

4. Hood DC, Bach M, Brigell M, Keating D, Kondo M, Lyons JS, Palmowski-Wolfe AM (2008) ISCEV guidelines for clinical multifocal electroretinography (2007 edition). Doc Ophthalmol 116:1-11. doi:10.1007/s10633-007-9089-2

5. Holder GE, Brigell MG, Hawlina M, Meigen T, Vaegan, Bach M (2007) ISCEV standard for clinical pattern electroretinography (2007 update). Doc Ophthalmol 114:111116. doi:10.1007/s10633-007-9053-1

6. Brown M, Marmor MF, Vaegan, Zrenner E, Brigell M, Bach M (2006) ISCEV Standard for clinical electro-oculography (EOG) 2006. Doc Ophthalmol 113:205-212. doi:10.1007/ s10633-006-9030-0

7. Odom JV, Bach M, Barber C, Brigell M, Holder G, Marmor MF, Tormene AP, Vaegan (2004) Visual evoked potentials standard. Doc Ophthalmol 108:115-123. doi:10.1023/B: DOOP.0000036790.67234.22 